\title{
Barre palatine : situations cliniques et analyse biomécanique
}

\section{RÉSUMÉ}

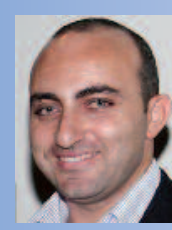

Hicham BENYAHIA

Professeur assistant

de l'enseignement supérieur

en orthopédie dento-faciale,

Faculté de médecine dentaire,

Université Mohamed V Souissi Rabat,

BP 6212, Rabat-Instituts,

Madinat Al Irfane,

Rabat, Maroc.

\section{Fatima ZAOUI}

Professeur d'enseignement supérieur en orthopédie dento-faciale,

Faculté de médecine dentaire,

Université Mohamed V Souissi Rabat.
La biomécanique orthodontique est l'étude de l'équilibre des systèmes de forces opérant en bouche. Notre propos porte sur les applications des situations biomécaniques d'équilibre dans les indications et activations de la barre palatine.

Elle peut être activée en clinique pour délivrer des forces et des moments utiles pour déplacer et/ou déroter les molaires maxillaires dans les trois plans de l'espace. En général, trois schémas d'activation sont décrits : $V$ symétrique, $V$ asymétrique et relation en escalier. Toutefois, les forces d'équilibre associées aux moments des couples créés peuvent être la source de surprises cliniques, il convient de les connaître pour mieux les maîtriser. 


\section{Introduction}

$>$

L'équilibre statique est régi par la troisième loi de Newton : "Toute action entraîne une réaction égale et opposée. »

Lorsqu'un arc est engagé dans les attaches, un équilibre s'établit automatiquement de luimême. Le praticien n'a donc aucun effort à faire pour le réaliser. En revanche, il se doit de reconnaître les forces et les moments mis en jeu lors de son établissement [1].

Et ceci devient plus clair, à partir de l'interprétation pratique de l'énoncé de la loi : " $L a$ somme algébrique de tous les éléments d'un système de forces doit être nulle » :

$$
\begin{aligned}
& \sum \vec{F}=0 \\
& \sum M=0
\end{aligned}
$$

Les sommes des forces horizontales, verticales et des moments doivent être nulles.

Conclusion pratique : quel que soit le type d'activation réalisée par notre système de force - force horizontale, verticale, torque -, il se produit toujours une réaction égale et opposée souvent indésirable, qu'il convient de définir et de contrôler.

\section{Situations d'équilibre}

En appliquant cette troisième loi de Newton à l'orthodontie, trois types de relations d'équilibre entre arcs et attaches sont définis :

- relation en "V symétrique " ;

- relation en « V asymétrique ";

- relation en « escalier » $[1,2,3]$.

Ces trois situations d'équilibre résument I'ensemble des relations qui peuvent exister entre arcs et attaches. Bien évidemment, ces relations sont très variables et dépendent de la malocclusion initiale, des courbures d'activation sur I'arc et de l'angulation des attaches [1, 4].

Si l'on ne considère que deux dents, ces relations sont simples à analyser. Elles deviennent fort complexes lorsque l'on considère toutes les dents concernées par un arc continu [1, 3].

On va se limiter aux cas où la valeur d'ancrage des deux unités dentaires est semblable (centre de résistance du système situé au milieu), vu notre modèle d'étude : la barre palatine [5].

\section{Situation de «V asymétrique»}

Le fil va présenter une courbure décentrée par rapport aux deux boîtiers A et B [1] (fig. 1a).

Le contact du fil avec la lumière des boîtiers va être à l'origine de la création de deux forces à la partie mésiale et distale de chaque boîtier.

Ces forces sont parallèles, de sens opposé et de même intensité ; elles forment donc un couple. Par ailleurs, l'angulation entre le fil et le bracket étant plus forte du côté de A, le couple CA sera donc plus important que le couple CB [5].

Par simplicité, on peut considérer le moment du couple le plus faible CB comme nul, car c'est la différence algébrique entre les deux moments qui importe.

D'après les lois de Newton, pour que le système de force ainsi créé soit en équilibre, il faut qu'en un point quelconque du système, la somme des forces et des moments soit nulle. Par commodité, nous choisirons ce point comme étant le centre de résistance du système Rs. 


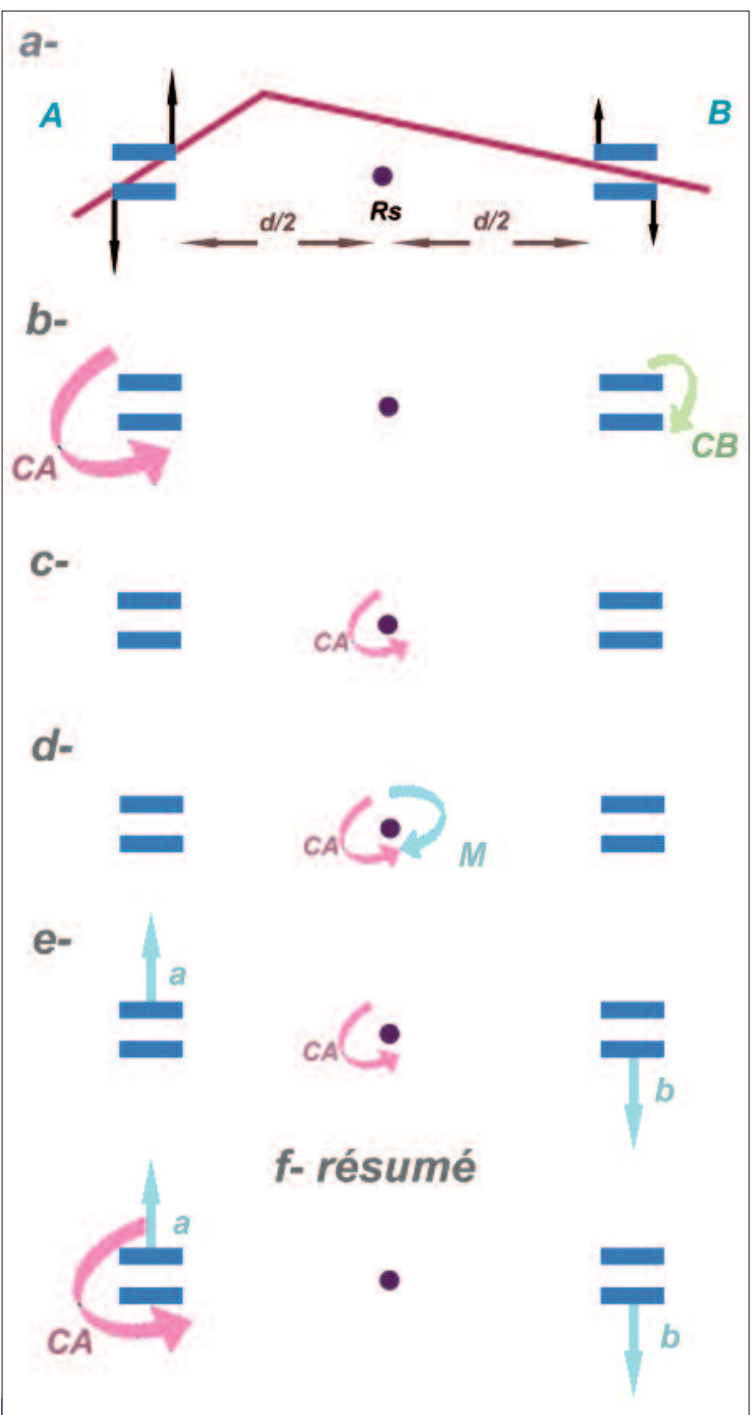

Fig. 1 a à $\mathrm{f}$ " Situation de « V asymétrique ».
Dans ce cas précis, l'équilibre du système nécessite l'existence d'un moment réactionnel $\mathrm{M}$ de sens opposé à CA et d'intensité égale (ou d'intensité égale à la différence en valeur absolue entre $\mathrm{CA}$ et $\mathrm{CB}$ ).

Pour visualiser les effets produits par $\mathrm{M}$ au niveau des deux dents $A$ et $B$, on peut décomposer le moment $M$ en deux forces $a$ et $b$ agissant respectivement sur $A$ et $B$.

On peut résumer ainsi le système en un moment sur $A$ et deux forces d'intensités égales et de sens opposés sur A et B (fig.1a-f).

II est utile de rappeler que ce schéma résumé peut s'appliquer aussi bien dans un plan frontal ( $a$ et $b$, forces d'ingression et d'égression) que dans un plan horizontal ( $a$ et $b$, forces mésialante et distalante).

\section{Situation de «V symétrique»}

Il s'agit en fait d'un cas particulier de la relation en $V$ asymétrique où la courbure se trouve centrée entre les deux boîtiers. Les moments de couple ainsi créés $C A$ et $C B$ sur chaque dent sont de même intensité mais de sens opposé. Au point Rs, les deux moments s'annulent et il n'y a pas création de force horizontale ou verticale. Il y aura seulement un mouvement de rotation de $A$ et $B$ en sens opposé $[2,3,5]$ (fig. 2).

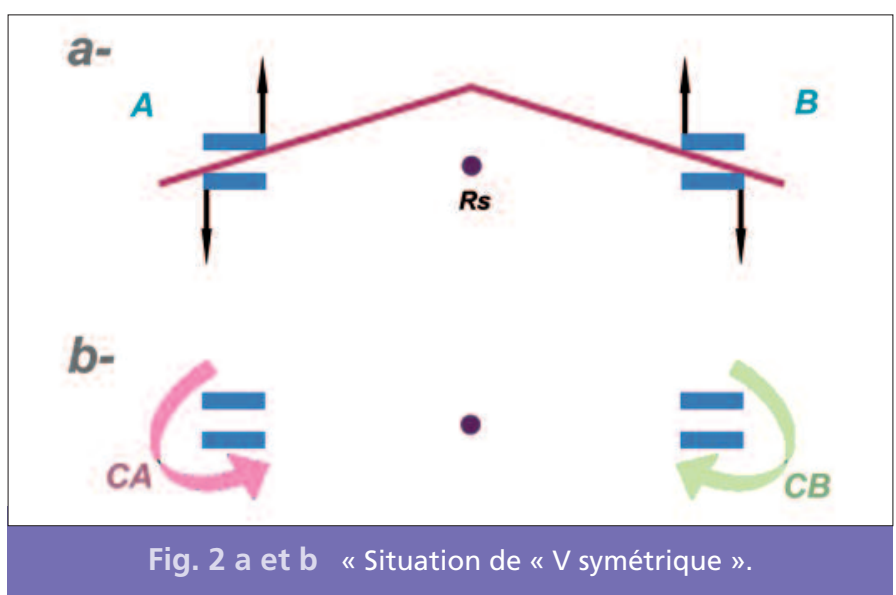




\section{Situation de}

\section{« relation en escalier»}

L'arc présente une double courbure. Sur chacun des boîtiers de $A$ et $B$, il y a création d'un couple. Les deux couples $C A$ et $C B$ sont de même intensité et de même sens. Au Rs, les deux moments de couples s'additionnent et il y a donc apparition d'un moment réactionnel $M$, de sens opposé et d'intensité double de CA ou de CB. Ce moment peut être décomposé au niveau de $A$ et $B$ en deux forces égales et opposées, a et $b$.

En résumé, on aura deux moments de même sens et de même intensité et deux forces opposées.

Les forces créées dans ce genre de situation sont plus importantes que dans la situation en $V$ asymétrique. En effet, les deux moments $C A$ et $C B$ s'additionnent dans la situation en escalier et le moment réactionnel est donc doublé, ainsi que l'intensité des forces générées (fig. 3).

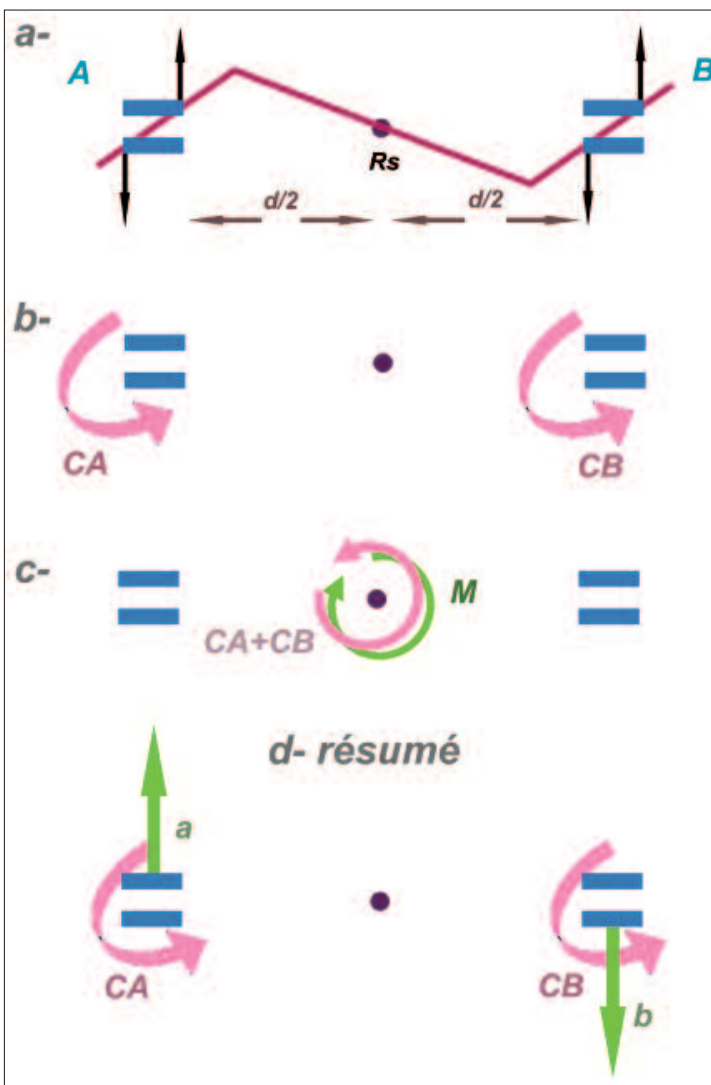

Fig. 3 a à d Situation de « relation en escalier ».

\section{Applications cliniques : barre palatine}

Le terme générique de barre palatine englobe un certain nombre d'appareillages et de dispositifs reliant les molaires droite et gauche : arc de Nance, arc transpalatin, arc Ghoshgarian, arc en forme de fer à cheval de Burstone, Quad'helix, arc palatin d'expansion en forme de $« \mathrm{~W}$ » de Burstone... [2, 3].

Ces appareillages présentent des caractéristiques biomécaniques similaires lors de leur activation et le système de forces développé est relativement simple. Toutefois, les indications cliniques nombreuses de la barre palatine en font un outil clinique incontournable.
Les indications principales d'activation de la barre palatine sont les suivantes:

- dérotation molaire : (arc transpalatin) ;

- action transversale sur la distance intermolaire

(expansion ou contraction) (Quad'hélix) ;

- torque molaire (arc transpalatin) ;

- recul molaire (arc transpalatin) $[6,7]$;

- ingression molaire (arc transpalatin) [8].

Il existe deux types de transpalatin : I'amovible inséré dans les fourreaux et le transpalatin soudé aux bagues molaires. Ce dernier trouve encore sa place dans notre pratique clinique, de par sa simplicité de réalisation, la possibilité de 
le prescrire même à un âge précoce, son efficacité intéressante, à condition d'être exigeant dans sa réalisation; notamment, au niveau du bras soudé du transpalatin qui doit être le plus long possible et couvrir la totalité de la face palatine de la molaire, afin que le contrôle de celle-ci soit maximum (fig. 4).

Le transpalatin amovible nécessite I'utilisation de bagues molaires à fourreaux palatins. Le scellement préalable de ces bagues permet à I'activation du transpalatin amovible d'être plus efficace sans perte d'informations (fig. 5).

Des bagues de dimensions moins adaptées aux molaires peuvent se déformer au moment du scellement du transpalatin soudé à cause des forces créées par l'activation ; une perte plus ou moins importante des informations de dérotation ou de torque est inévitable.

Avant chaque activation, il faut vérifier la passivité de l'arc. Pour cela, il convient de réaliser une lecture en bouche (read-out), dans les trois sens de l'espace : horizontal, sagittal et vertical. Le read-out se fait en insérant un côté et en observant l'effet au niveau de l'autre côté qui doit être nul et réciproquement.

Il faut à chaque fois rechercher cette position de passivité ou de neutralité de l'arc, pour éviter d'avoir des effets parasites dont on ne soupçonne pas l'existence.

La boucle en $U$ du transpalatin peut être antérieure ou postérieure. Cette dernière position est plus profitable (fig. 5), d'une part pour un
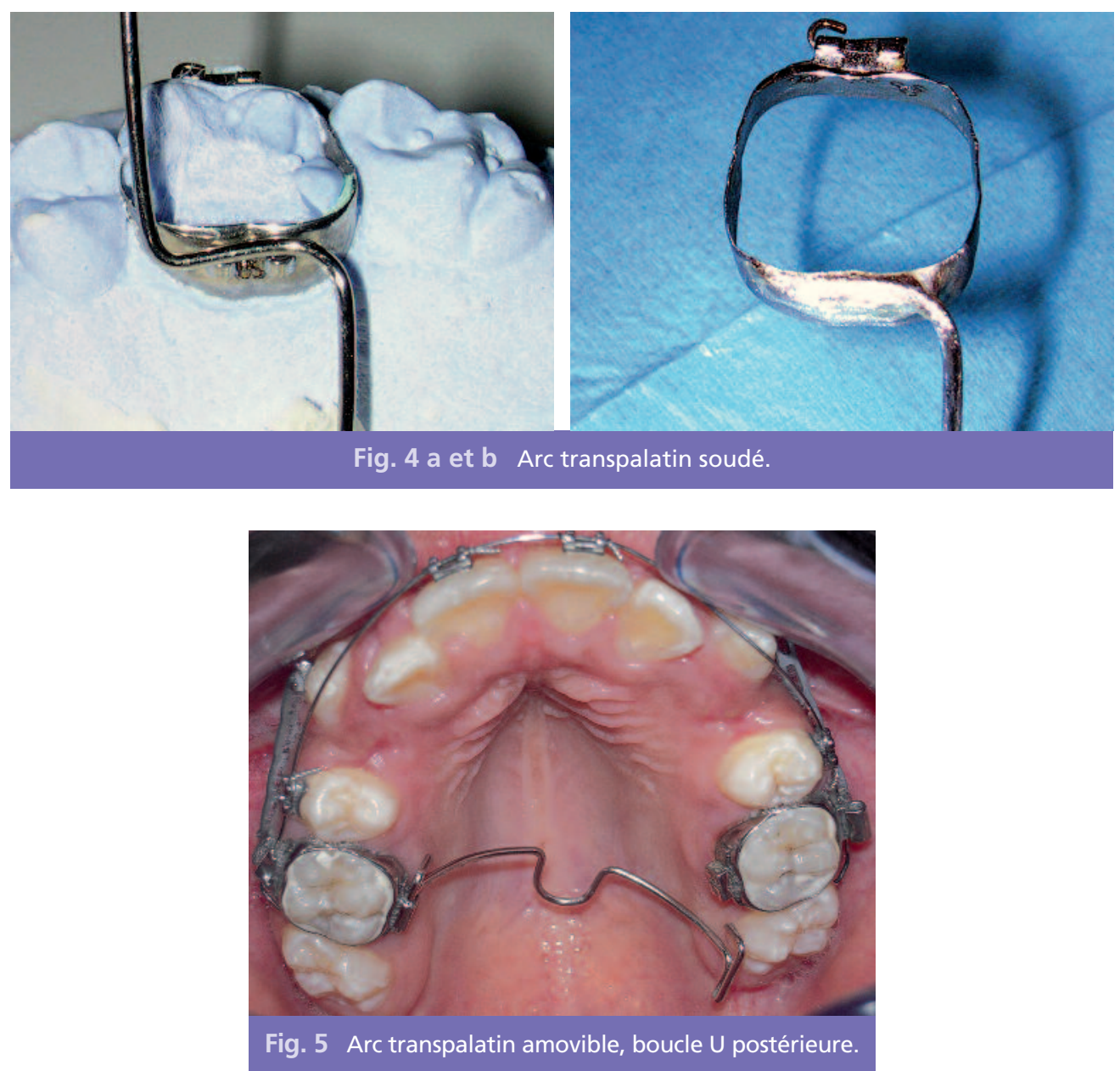
meilleur contrôle vertical par les appuis linguaux [8] et d'autre part pour plus d'efficacité et moins de perte d'activation, étant donné que la boucle va travailler en ouverture et non pas en fermeture (position antérieure).

\section{Expansion \\ et contraction bilatérales}

L'expansion et la contraction sont indiquées respectivement dans les cas d'exo-alvéolie ou d'endo-alvéolie maxillaire. Ces deux mouvements nécessitent une grande élasticité de l'appareil ; de ce fait, le quad'helix ou le bi-helix seraient plus appropriés, quoiqu'on puisse aussi indiquer un arc transpalatin dans des cas de faible décalage horizontal intermolaire.

L'expansion bilatérale est obtenue en augmentant la distance entre les deux bagues molaires. Cette force horizontale d'expansion, passant à distance du centre de résistance (C.res) de chaque molaire, va entraîner un mouvement de version corono-vestibulaire, avec un déplacement vestibulaire du centre de résistance $[2,7]$ (fig. 6).

La contraction bilatérale est obtenue en réduisant la distance entre les deux bagues molaires. Cette force horizontale de contraction, passant à distance du C.res de chaque molaire, va entraîner un mouvement de version corono-palatine, avec un déplacement palatin du C.res $[2,7]$ (fig. 7).

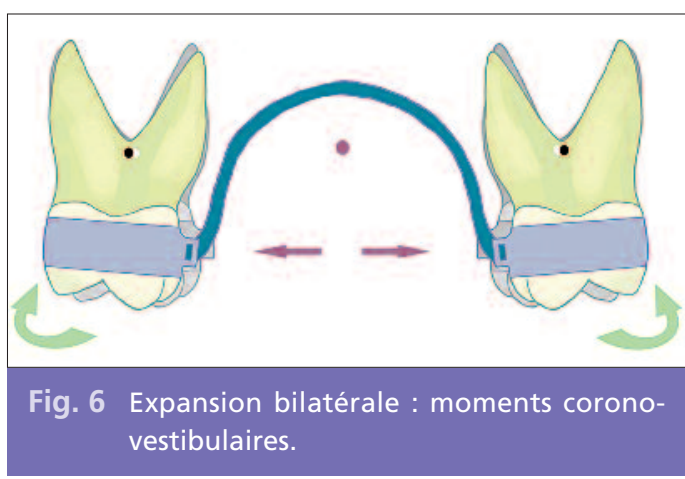

Le système de force développé dans ces deux activations représente une situation particulière différente du "V symétrique ", étant donné la présence au C.res de chaque molaire d'une force horizontale (d'expansion ou de contraction) associée à un moment (moment de force) ; ce dernier est la conséquence du passage de la ligne d'action de la force à distance du C.res de chaque dent. L'intensité de ce moment est égale au produit de la force horizontale d'expansion ou de contraction « $F$ » multiplié par la distance perpendiculaire $d$ séparant ligne d'action de la force au C.res [7].

$$
M=F x d
$$

L'association d'un torque adapté (cf. « Torque molaire " ci-après) à l'activation d'expansion ou de contraction permet un déplacement molaire, en gression, moins récidivant que la version [7].

\section{Correction des rotations molaires}

Du fait de la forme trapézoïdale de la molaire, sa dérotation permet un gain d'espace jusqu'à $2 \mathrm{~mm}$ par côté et une solution intéressante pour les classes II occlusales [9] (fig. 8).

\section{> Dérotation bilatérale (rotation mésio-vestibulaire)}

Activation de dérotation : saisir le joint de soudure, avec une pince Weingart, pour réaliser

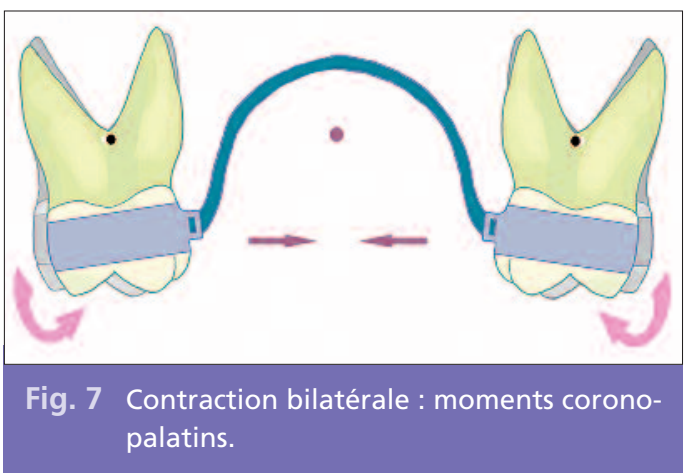




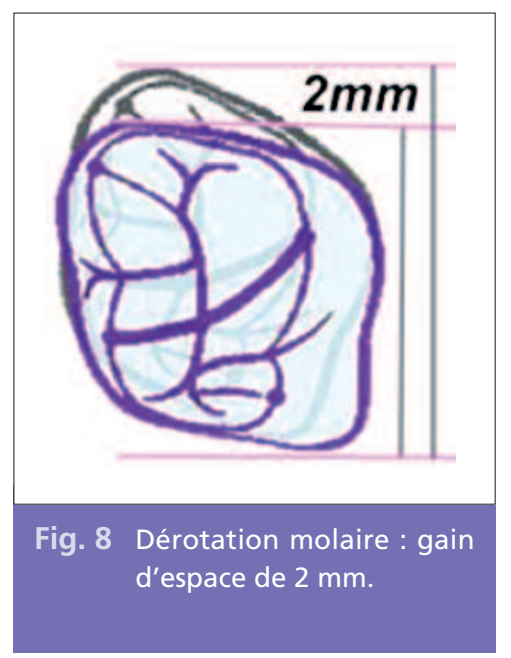

une plicature de toe-in qui doit être bilatérale et symétrique (fig. 9).

Transpalatin amovible : I'activation en dérotation peut se faire de la même façon par simple plicature de toe-in des bras destinés aux fourreaux.

La symétrie peut être vérifiée à l'aide d'une charte, ou par un read-out en bouche en deux temps (bague droite insérée et bague gauche en read-out et inversement) $[2,4,7]$.

II s'agit d'une situation de " V symétrique » au niveau du plan horizontal : existence de deux moments de couple d'égale intensité et de sens opposé qui vont exprimer un mouvement de rotation des molaires autour de leur C.res. (C.rot $=$ C.res) (fig. 10, 11 et 12).

À cause de la rigidité du transpalatin qui maintient la distance intermolaire (celle-ci est légèrement réduite par la plicature de toe-in), on aura un déplacement palatin du centre de rotation (C.rot). De ce fait, une légère expansion du transpalatin est nécessaire pour éviter un articulé inversé de la cuspide molaire disto-vestibulaire $[7,10]$.

Un gain d'espace important ne peut être obtenu qu'en dérotant la molaire autour du centre de l'attache linguale (fourreau ou soudure), et non pas autour du C.res de la molaire ;

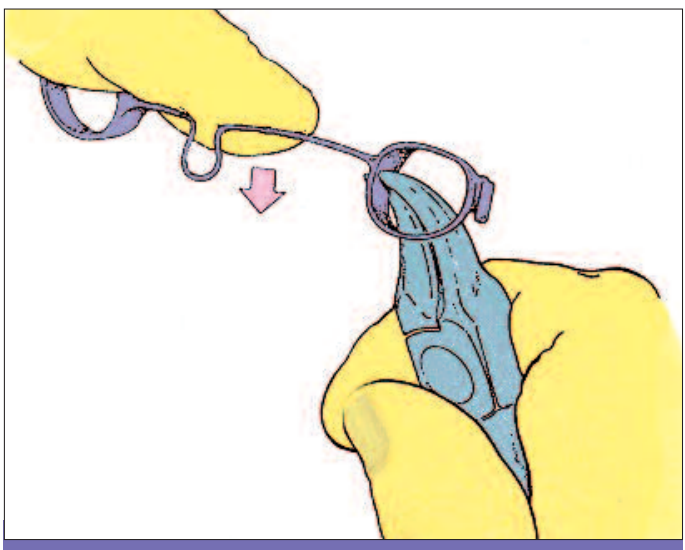

Fig. 9 Activation pour dérotation molaire d'un transpalatin soudé.

cela peut être obtenu par l'association d'une force extraorale au niveau du tube vestibulaire. L'étude de Braun S. et al. [11] confirme l'efficacité de cette association : une rotation maximale de $18^{\circ}$ est possible et permet un gain d'espace jusqu'à $2,1 \mathrm{~mm}$.

\section{Cas de classe II molaire thérapeutique :}

La classe II molaire thérapeutique requiert une finition particulière entre molaire supérieure et inférieure, particulièrement la molaire supérieure qui doit présenter une rotation mésiolinguale propice à la stabilité occlusale ; l'activation du transpalatin se fera par la réalisation de plicature " toe-out » bilatérale symétrique,

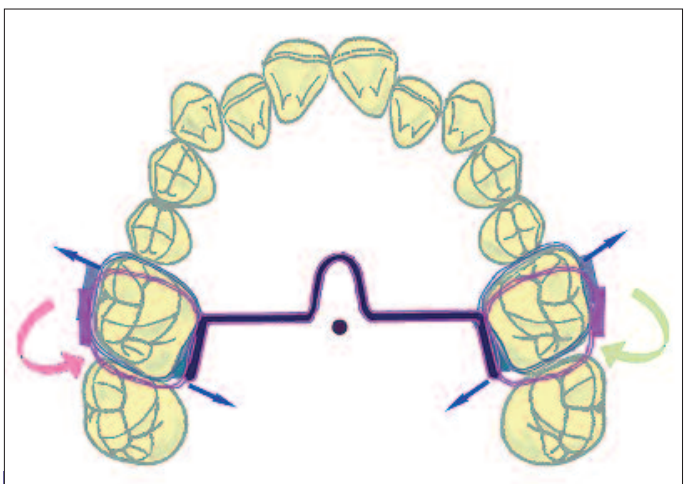

Fig. 10 Dérotation bilatérale : situation de « V symétrique ». 

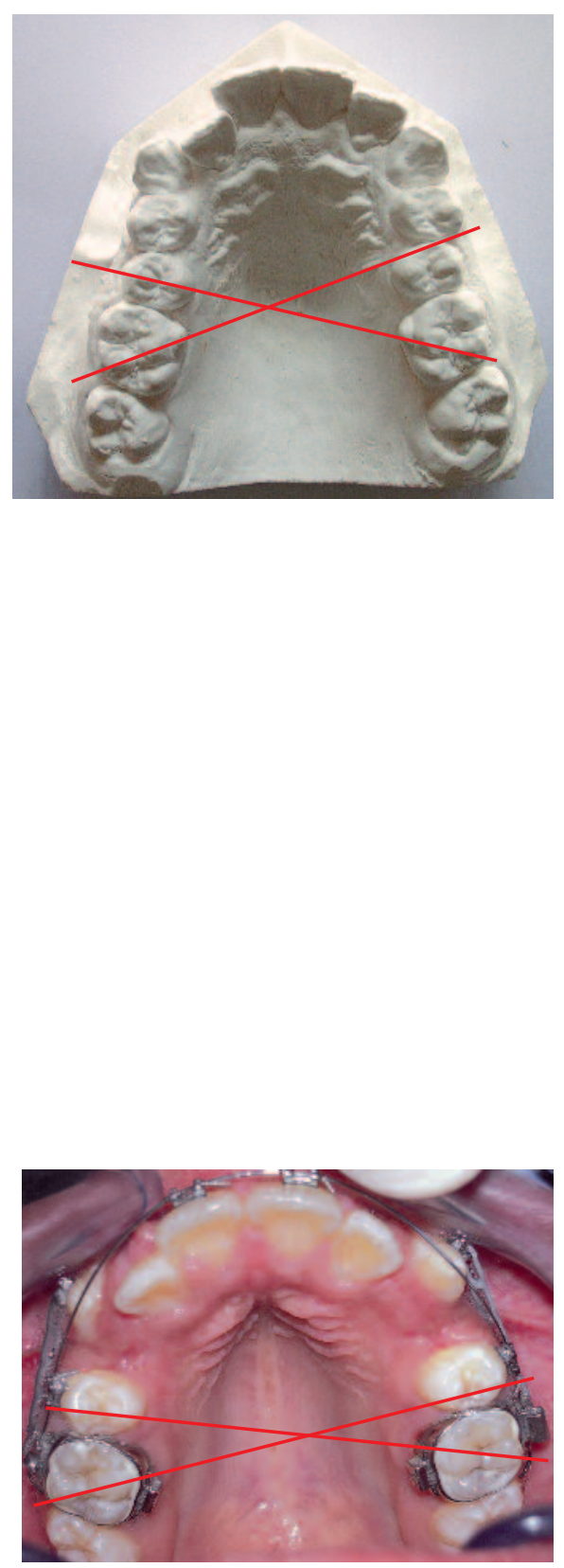
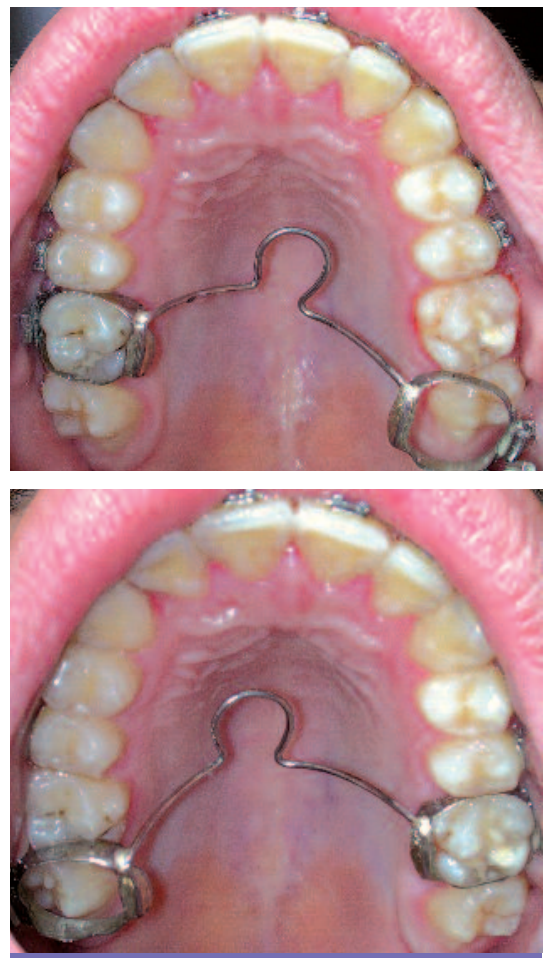

Fig. 11 a à d Cas d'endoalvéolie avec rotation importante des $1^{\text {res }}$ molaires ; arc transpalatin, read-out et dérotation symétrique.
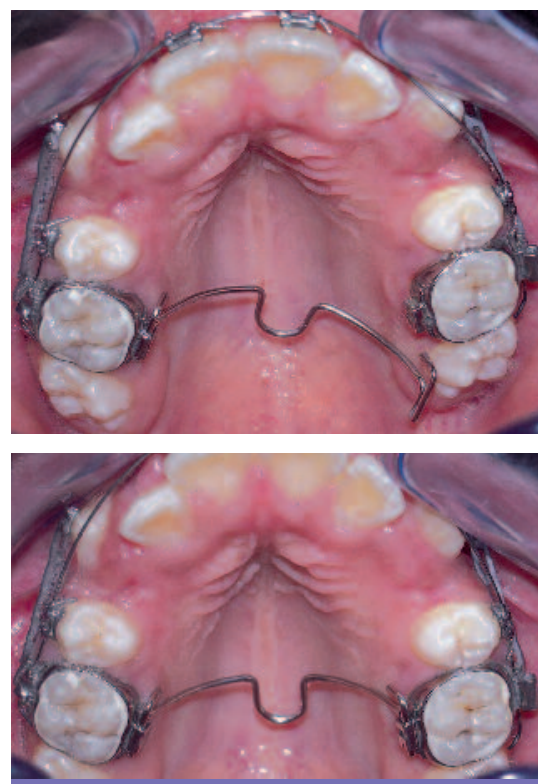

Fig. 12 a à $\mathrm{d}$ Rotation des $1^{\text {res }}$ molaires maxillaires ; arc transpalatin amovible, read-out et dérotation symétrique.
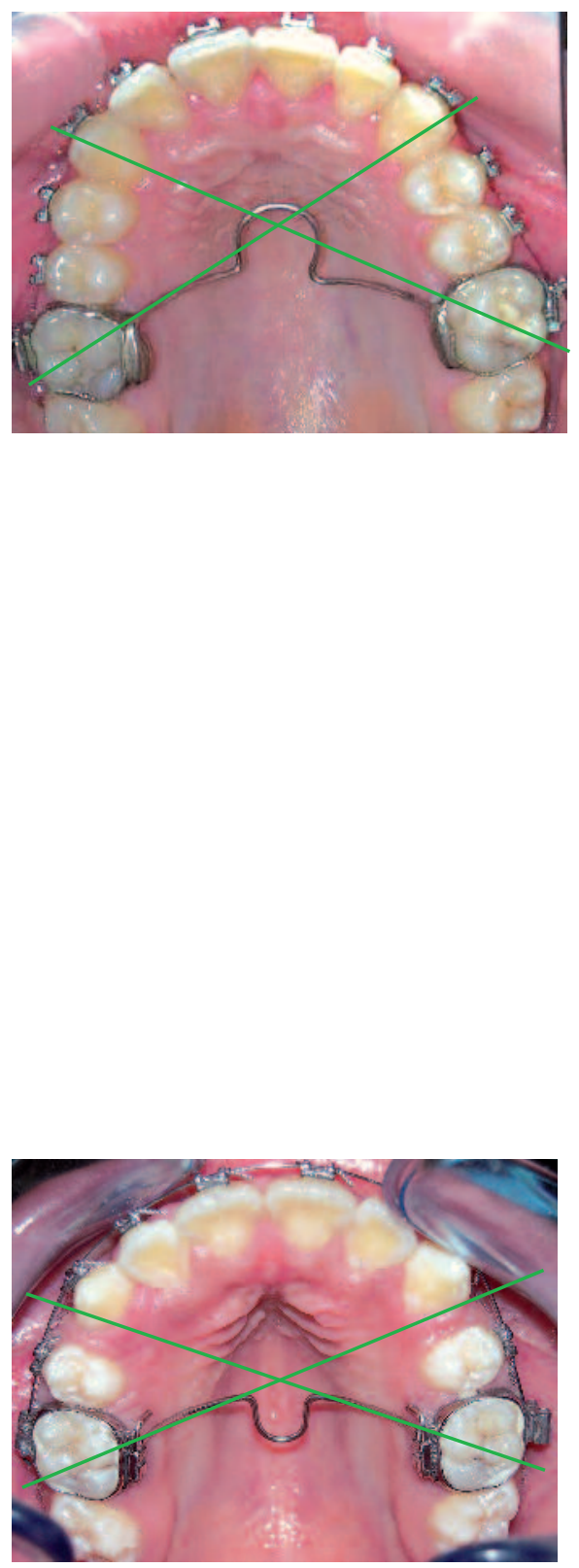
avec des moments égaux et opposés à ceux du schéma précédent [7].

\section{> Dérotation unilatérale (rotation mésio-vestibulaire)}

L'activation va se faire d'un seul côté au niveau de la molaire en rotation, par une plicature de toe-in au niveau du joint homolatéral. L'équilibre du système va nécessiter des forces horizontales : mésiale sur la molaire à déroter et distale sur la molaire opposée.
Le read-out en bouche objective la disto-position de la bague opposée (fig. 13).

Le moment de dérotation ainsi que les forces développées peuvent être quantifiés aisément, grâce au read-out (fig. 14). Un dynamomètre va mesurer la force nécessaire $F$ pour replacer la bague sur la molaire controlatérale.

Le moment de dérotation $M$ est égal au produit de la force multipliée par la distance $d$ séparant les deux joints. $M=F \times d$.
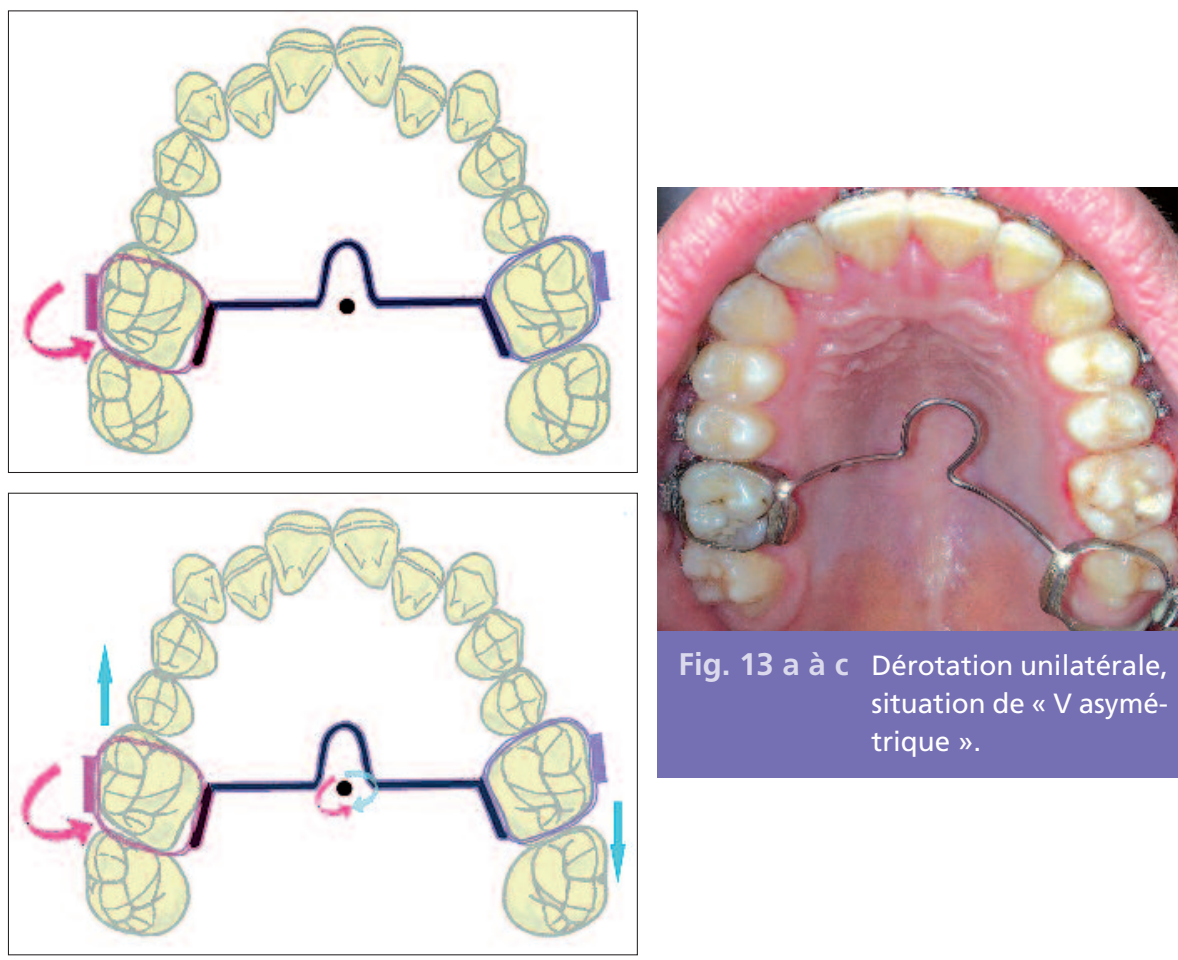

Fig. 13 a à c Dérotation unilatérale, situation de « V asymétrique $»$.

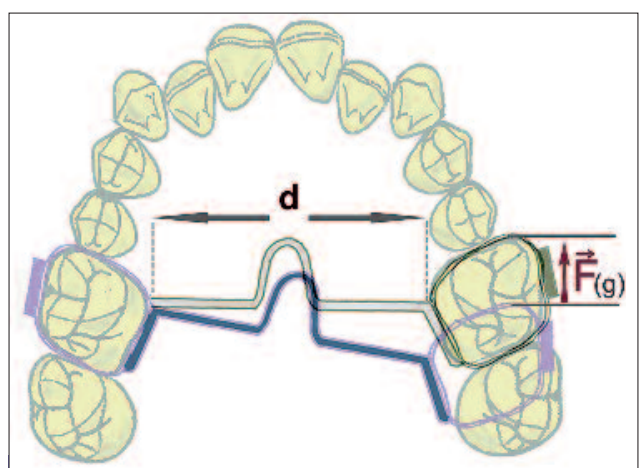

Fig. 14 Read-out en dérotation unilatérale. 
Les forces mésiale et distale créées par le moment réactionnel sont égales à $F$.

Contrecarrer la force mésiale et donc la dérive mésiale de la molaire homolatérale, par un dispositif intra- ou extra-oral, permet de renforcer la force distale qui double d'intensité $2 F$. Ce système de force peut également trouver son indication dans les cas de recul molaire unilatéral.

De façon similaire, ce système de force peut également être indiqué dans les cas de fermeture d'espaces résiduels situés en mésial de la première molaire $[7,10]$.

\section{Torque molaire}

L'activation du transpalatin peut se faire de façon bilatérale ou unilatérale.

L'activation se fait dans le plan frontal par la réalisation d'une pliure interne du joint (fig. 15).

\section{$>$ Torque bilatéral (torque radiculo-vestibulaire)}

Indiqué en présence d'une version corono-vestibulaire des molaires, en association avec l'expansion symétrique pour obtenir un mouvement de gression ou encore pour rechercher un ancrage cortical $[2,6]$.

Il s'agit d'une situation de "V symétrique » avec deux moments de rotation dans le plan frontal $[2,9]$ (fig. 16).
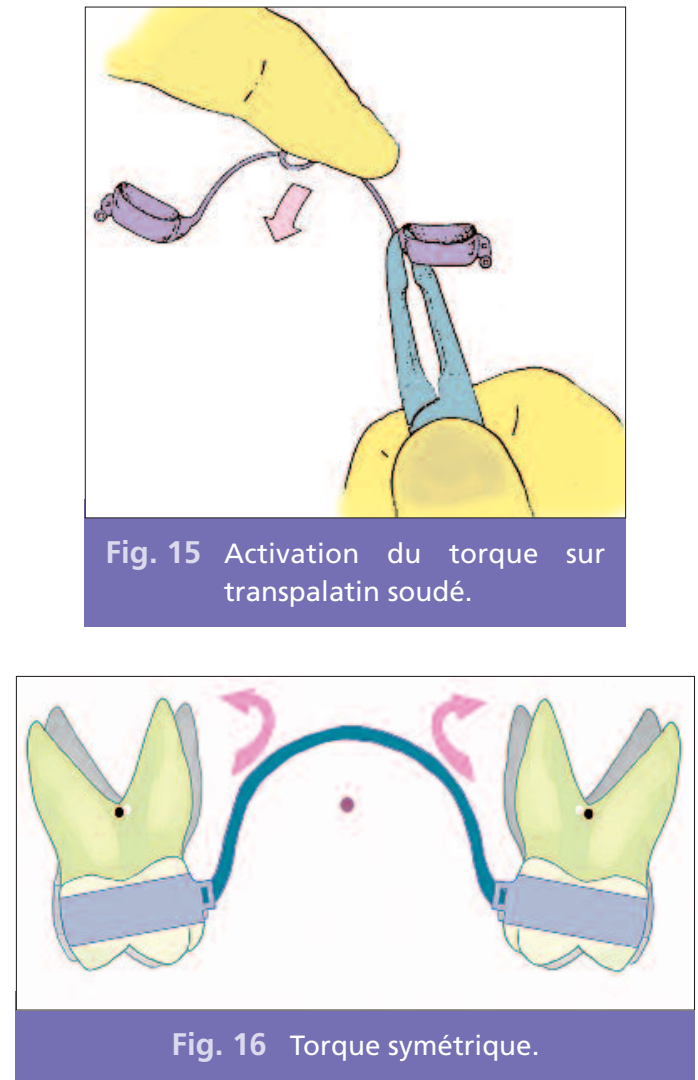

La rigidité de l'arc transpalatin impose une force vestibulaire qui agit au niveau coronaire ; on aura donc plus de mouvement radiculaire, avec déplacement du C.res en direction vestibulaire. Le C.rot se trouve déplacé occlusalement $[7,12,13]$.

Le read-out en bouche se fait en deux temps, la bague opposée se situe occlusalement par rap-

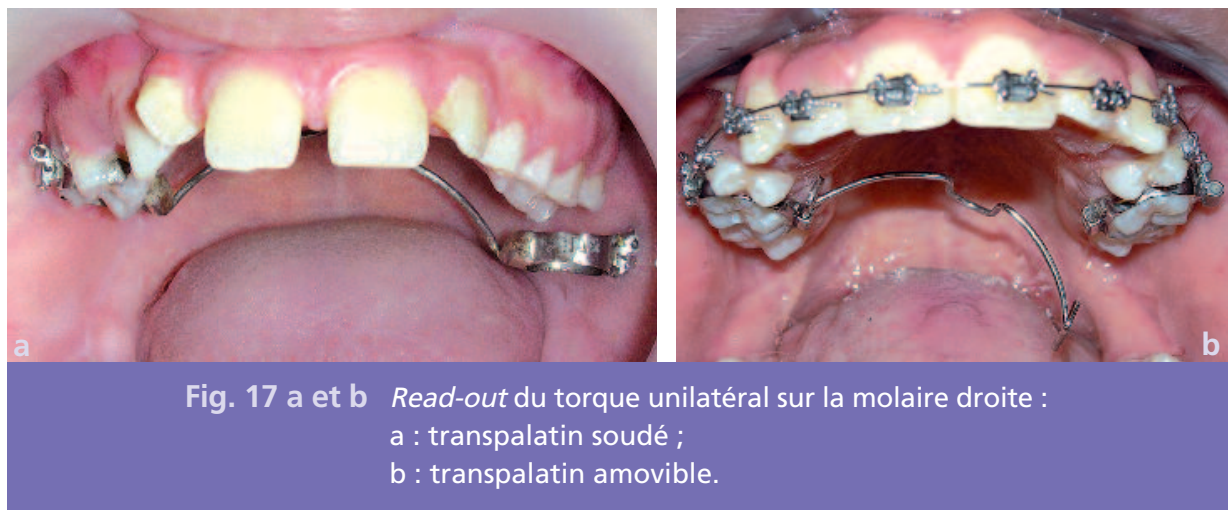


port à la table occlusale de la molaire [2] (fig. 17).

La quantification du moment de torque peut se faire à l'aide d'un dynamomètre.

$>$ Torque unilatéral

(torque radiculo-vestibulaire)

L'activation unilatérale comme dans le cas de dérotation asymétrique sera à l'origine d'un moment de couple (torque radiculo-vestibulaire), engendrant un moment réactionnel équilibrant le système avec deux forces verticales dans le plan frontal $[7,10]$, une force ingressive sur la molaire homolatérale et égressive sur la molaire controlatérale (fig. 18).

En clinique, les forces musculaires et l'occlusion vont empêcher l'égression molaire, et le plus souvent on remarque l'ingression et l'intrusion de la molaire sujette au torque unilatéral.

\section{> Torque unilatéral avec expansion}

Le torque radiculo-vestibulaire unilatéral associé à une expansion du transpalatin peut être

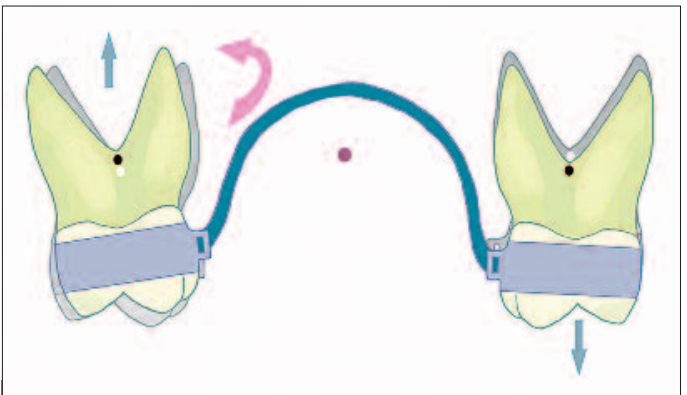

Fig. 18 Torque asymétrique situation de « V asymétrique ».

indiqué dans les cas d'articulé inversé unilatéral ; l'activation du torque radiculo-vestibulaire ou corono-palatin sur la molaire en normoclusion va contrecarrer l'effet d'expansion à son niveau pour le majorer au niveau de la molaire controlatérale à déplacer vestibulairement.

\section{> Torque unilatéral avec contraction}

Le torque radiculo-palatin ou corono-vestibulaire unilatéral associé à une contraction du transpalatin peut être indiqué dans les cas
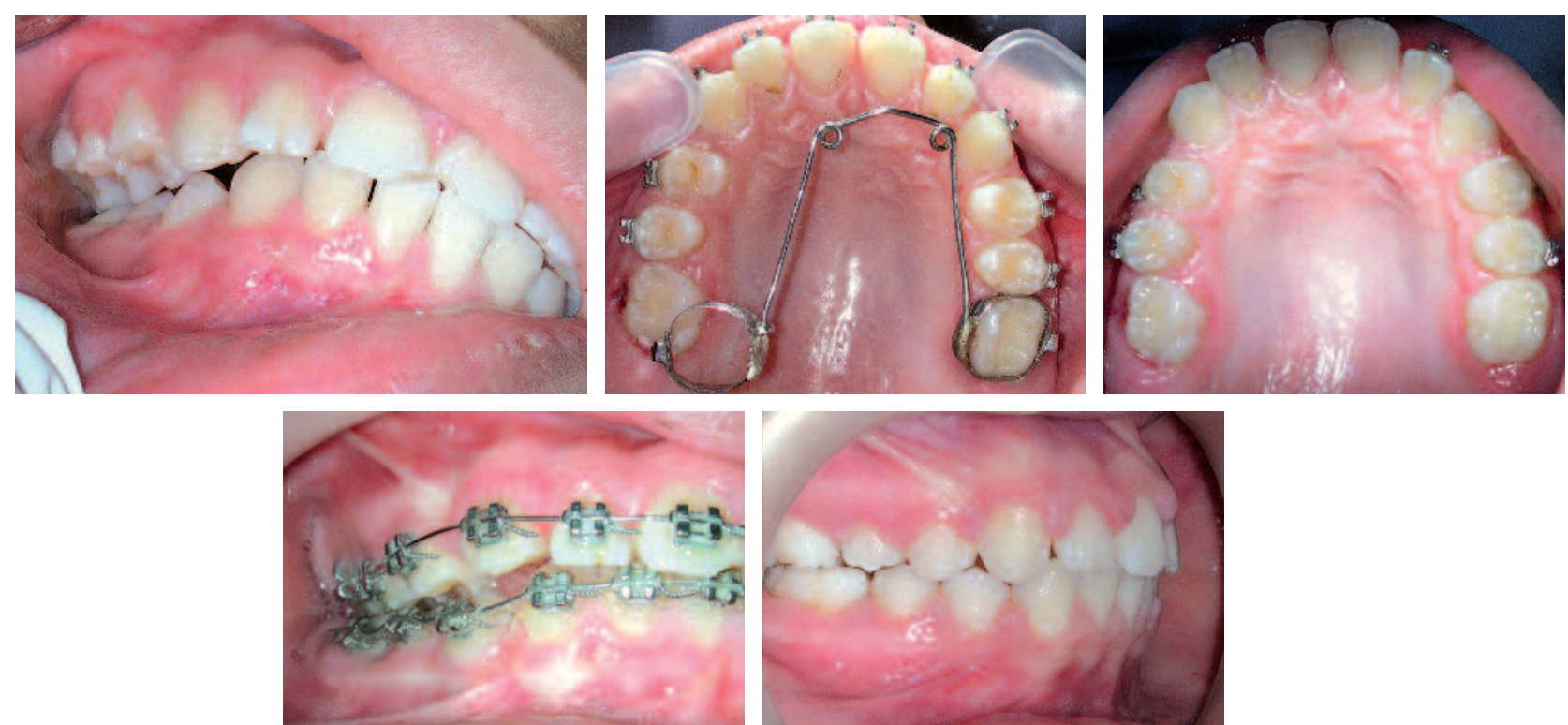

Fig. 19 a à e Cas d'exoalvéolie maxillaire droite ; bi-helix en contraction avec torque corono-vestibulaire sur la 26 (à noter l'ingression de 16 en réaction) ; cas en fin de traitement. 
d'exoalvéolie unilatérale ; l'activation du torque radiculo-palatin ou corono-vestibulaire sur la molaire en normoclusion va contrecarrer I'effet de contraction à son niveau pour le majorer au niveau de la molaire controlatérale à déplacer en direction palatine [7] (fig. 19).

\section{Distalisation molaire}

Le transpalatin peut être indiqué dans les cas de recul asymétrique des molaires, on a vu précédemment l'activation en " V asymétrique " dans la dérotation unilatérale.

L'activation de "relation en escalier " peut aussi être indiquée à ce propos, obtenue par la réalisation de deux plicatures différentes : une plicature de toe-out sur la molaire à distaler, et une plicature de toe-in sur la molaire controlatérale.
Les forces horizontales générées vont être plus importantes que dans la situation de " $\mathrm{V}$ asymétrique " (fig. 20).

Il convient néanmoins de contrôler la force mésiale par l'adjonction d'une force extra-

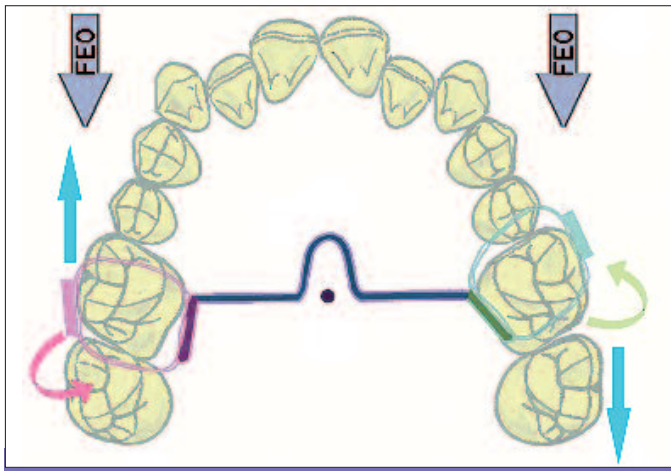

Fig. 20 Situation de " relation en escalier » associée aux forces extra-orales (FEO).
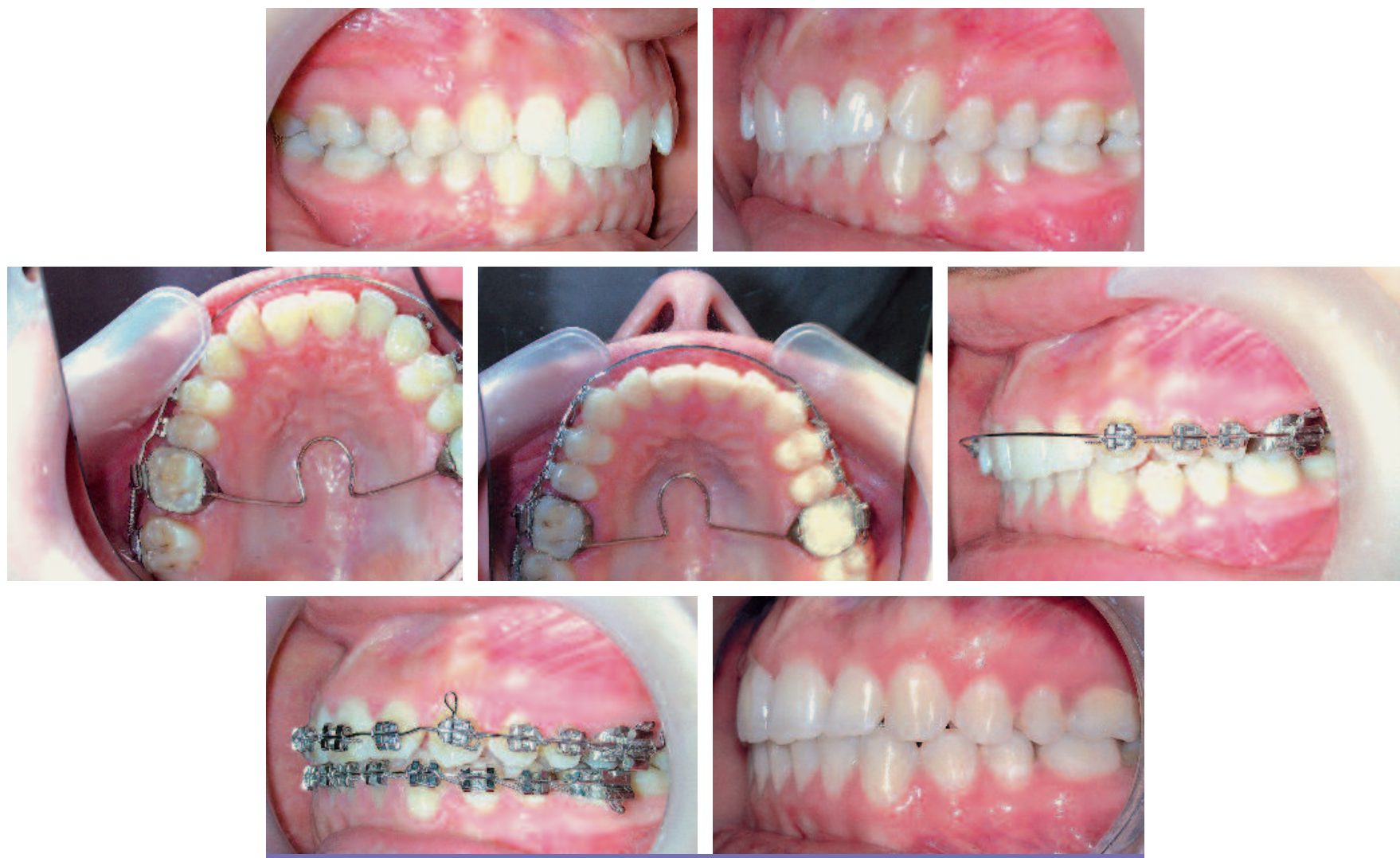

Fig. 21 a à g Distalisation molaire unilatérale dans un cas de classe II, subdivision gauche. 
orale [7], ou l'utilisation d'une minivis d'ancrage. II est à noter également qu'une force extra-orale seule n'est pas indiquée pour un recul molaire unilatéral. Les forces distalantes générées seront symétriques et vont déstabiliser la classe I molaire précieuse du côté normal. Le transpalatin n'a pas son égal pour orienter le système de force du côté désiré (fig. 21).

$N B$ : I'activation en " relation d'escalier " peut être indiquée dans toutes les situations asymétriques : dérotation unilatérale, torque unilatéral, recul molaire unilatéral, lorsqu'on requiert une intensité de forces plus importante [7].

Toutefois, il ne faut pas exagérer les plicatures (5 à $10^{\circ} \mathrm{d}$ 'inclinaison est suffisant) sinon on aura une difficulté à insérer le transpalatin.

\section{Ingression molaire}

Le transpalatin peut être indiqué dans le contrôle du sens vertical en cours de croissance. La boucle médiane du transpalatin avec ou sans pastille en résine (fig. 22a) et à distance de la fibromuqueuse du palais d'au moins $2 \mathrm{~mm}$ percevrait des forces linguales ingressives $[7,8]$.

En effet, le contact itératif du dos de la langue contre cette boucle au moment de la déglutition et de la mastication favorise l'ingression et lutte contre l'égression molaire maxillaire responsable de l'augmentation de la divergence faciale.

L'association minivis palatine et arc transpalatin est intéressante pour l'ingression molaire, et le traitement des béances squelettiques chez les hyperdivergents, tout en maintenant le sens transversal (fig. 22b).

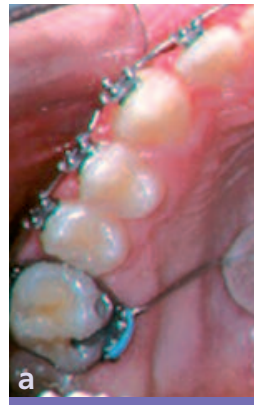

Fig. 22 a et $b$
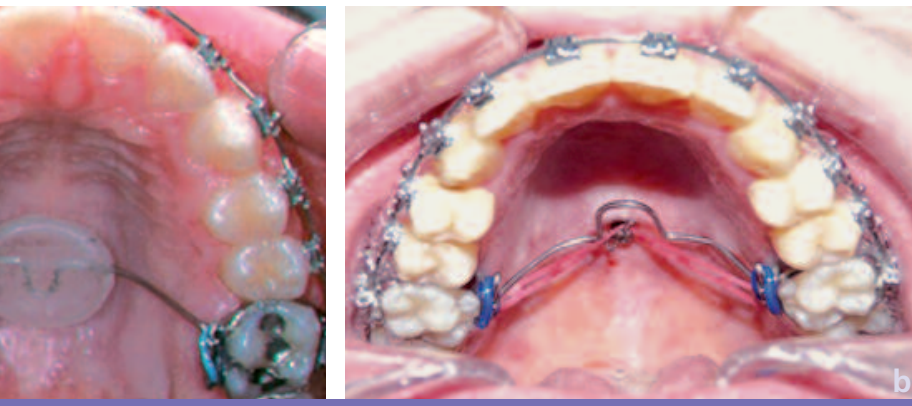

Contrôle du sens vertical. a : boucle médiane et pastille en résine à distance du palais ; $b$ : minivis palatine pour ingression molaire.

\section{Conclusion}

L'arc transpalatin demeure un outil incontournable en clinique orthodontique. Son efficacité n'est plus à démontrer pour des indications quasi systématiques, notamment la dérotation, le torque, le recul molaire. Toutefois, une bonne connaissance des principes biomécaniques, particulièrement des situations d'équilibre, est nécessaire pour une prescription et une activation judicieuses et efficaces de cet appareil.

Même avec la révolution amenée par les minivis d'ancrage, le contrôle de la dimension transversale des arcades dentaires notamment maxillaire reste aléatoire et complique la maîtrise des deux autres sens sagittal et vertical. 
La prise de conscience de l'importance du sens transversal vis-à-vis des autres sens devrait réha- biliter la place du transpalatin en clinique orthodontique.

\section{Bibliographie}

1. Mulligan TF. (traduit et synthétisé par Flageul F.).

Du bon usage de la mécanique en orthodontie. Rev Orthop Dento Faciale 1982;16(3):299-305 ; $1982 ; 16(4): 483-96$; 1983;17(1):67-79 ; 1983;17(2):299-305.

2. Nguyen-Gauffre MA. Etude mécanique des activations d'un arc transpalatin dans le plan occlusal. Mémoire CECSMO : Paris V, 1997.

3. Nguyen-Gauffre MA, Decker A, Augerau D, Pierrisnard L, Barquins M. Utilisations cliniques de la barre palatine. J Edg 1999;40:9-29.

4. Mulligan TF. Molar control. Part 1. J Clin Orthod 2002;36(1);11-23.

5. Demange $C$. Les situations d'équilibre dans les systèmes de force. Am J Orthod Dentofacial Orthop (ed. Fr.) 1990;3(3):118-24.

6. Burstone $\mathrm{CJ}$. Precision lingual arches. Active applications. J Clin Orthod 1989;23(2):101-9.

7. Rebelatto J.

Two-couple orthodontic appliance systems: transpalatal arches. Semin Orthod 1995; 1(1);44-54.

8. Chiba $Y$, Motoyoshi M, Namura $S$. Tongue pressure on loop of transpalatal arch during deglutition. Am J Orthod Dentofacial Orthop 2003;123:29-34.

9. Shroff B, Nanda R. Biomechanics of Class II correction. In: Nanda R. Biomechanics in clinical orthodontics. Philadelphia: WB. Saunders Co, 1997:143-155.
10. Proffit WR, Fields HW. Biomechanics and mechanics. In: Proffit WR, Fields HW (ed.). Contemporary orthodontics (3rd ed.). St Louis: Mosby, 2000;295-361.

11. Braun $S$, Kusnoto $B$, Evans $C A$. The effect of maxillary first molar dérotation on arch length. Am J Orthod Dentofacial Orthop 1997;112(5):538-44.

12. Baldini $\mathrm{G}$. Interaction du torque radiculo-vestibulaire et de l'expansion de l'arc palatin de Goshgarian. Rev Orthop Dento Faciale 1983;17(1):81-87.

13. Baldini G. Mesure expérimentale des moments de rotation dus au torque de l'arc palatin et de la force d'expansion résultant de ce torque. Rev. Orthop Dento Faciale 1983;17(2):243-54.

\section{SUMMARY}

\section{Palatal bar: clinical situations and biomechanical analysis}

Hicham BENYAHIA, Fatima ZAOUI

\section{Keywords \\ - palatal bar \\ - biomechanics \\ - equilibrium situations}

Orthodontic Biomechanics is the study of equilibrium of force delivering systems operating in the mouth. Our concerns about the applications of equilibrium biomechanic situations in indications and activations of the palatal bar.

It can be activated to deliver a clinically useful forces and couples to move and/or rotate maxillary molars in all three planes of space.

In general, three activation patterns are described: symmetrical V-bends, asymmetrical V-bends, and step bends. However, the associated equilibrium forces of created couples can be the source of clinical surprises, they should be better known to master. 


\section{Questions-Réponses}

> 1. Avant chaque activation du transpalatin, il est recommandé de vérifier la passivité de l'arc dans les trois sens de l'espace, comment?

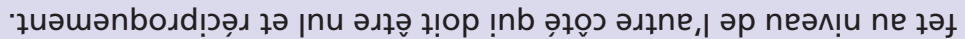

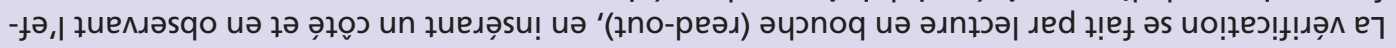

$$
\text { : әsuodə̧y }
$$

> 2. Pourquoi recherche-t-on cette position de passivité ou de neutralité de l'arc ?

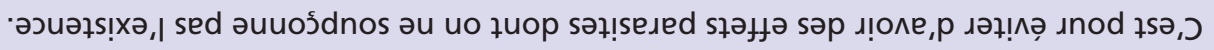

$$
\text { : əsuodə⿳亠口冋 }
$$

3. Quel type de mouvement est observé au niveau des molaires, après l'activation d'expansion? Donner une explication biomécanique.

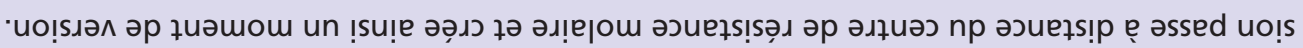

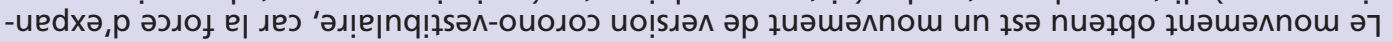

$$
\text { : əsuodə̧y }
$$

4. Pour obtenir une expansion maxillaire (molaire) symétrique en gression, que faut-il associer à l'activation d'expansion ?

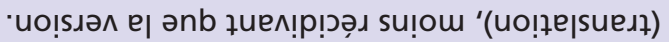

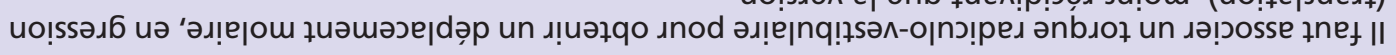

$$
\text { : әsuodə⿳亠二口犬 }
$$

5. Que montre le read-out en bouche d'une activation de dérotation molaire unilatérale?

·əə̧⿻コ一

: əsuodə̧y 